\title{
Melatonin and postoperative delirium: a possible link?
}

\author{
Farhanah Yousaf, MBBS · Edwin Seet, MBBS • \\ Lashmi Venkatraghavan, MD • Rita Katznelson, MD • \\ Frances Chung, MD
}

Received: 8 February 2010/Accepted: 17 May 2010/Published online: 4 June 2010

(C) Canadian Anesthesiologists' Society 2010

\section{To the Editor,}

Melatonin, an indole neurohormone, is the principal secretory product of the pineal gland that is synthesized from tryptophan and released directly into the bloodstream in a circadian pattern governed by the light and dark cycle. It produces hypnotic effects by accelerating sleep initiation and improving sleep maintenance and efficiency. Unlike most hypnotics, melatonin is not associated with significant changes in sleep architecture, hangover effects, substance dependence, or the potential for substance abuse. In addition, melatonin possesses the capacity to resynchronize the human circadian rhythm, and it may play a role in physiological adaptation to jet lag and shift work. Considering its hypnotic and chronobiotic properties, melatonin may also serve a role in the perioperative setting when the patient's sleeping patterns are likely to be disturbed. Indeed, the literature reveals an altered pattern of melatonin secretion after anesthesia and surgery. ${ }^{1}$ It is possible that pathological variation in melatonin levels or a disturbed circadian rhythm may modify the predisposition, severity, course, or outcome of postoperative complications, especially postoperative delirium, which is multifactorial.

The circadian rhythm of melatonin secretion has shown to be altered postoperatively, and this alone may lead to changes in sleeping patterns. ${ }^{1}$ Delirium and perioperative sleep disturbance are potentially linked - either as predisposing factors or as symptoms of one another. Thus, restoring normal sleeping patterns with a substance that is

F. Yousaf, MBBS - E. Seet, MBBS - L. Venkatraghavan, MD .

R. Katznelson, MD · F. Chung, MD ( $\square)$

University Health Network, University of Toronto,

Toronto, ON, Canada

e-mail: frances.chung@uhn.on.ca capable of resynchronizing circadian rhythm may possibly reduce the incidence of postoperative delirium or improve its outcome.

The authors of the first study to link melatonin and delirium investigated hormone synthesis during alcohol withdrawal, and they noticed an irregular rhythm of hormone secretion during delirium tremens. ${ }^{2}$ Also, there is some limited evidence that abnormal melatonin secretion in the perioperative setting is associated with a greater incidence of delirium. Shigeta et al. observed two different abnormal patterns of melatonin secretion in a small study of elderly surgical patients experiencing postoperative delirium. ${ }^{3}$ Postoperative melatonin concentrations were lower than preoperative serum concentrations when delirium developed as a single complication, and they were higher when delirium occurred in the presence of other complications in the postoperative period. An altered melatonin secretory pattern was also observed in 41 patients scheduled for esophagectomy. ${ }^{4}$ A reduced frequency of peak melatonin serum concentrations was apparent in $73 \%$ of patients who developed delirium in the postoperative period. $^{4}$

In addition, during the acute and recovery phases, melatonin concentrations were seen to vary according to the clinical subtypes of delirium, i.e., hyperactive, hypoactive, and mixed forms. ${ }^{5}$ Higher urinary 6-sulfatoxymelatonin excretion was observed in the hypoactive group of delirious patients, whereas it was significantly lower in the hyperactive group. No difference was detected in the mixed group of delirious patients during the acute and recovery phases.

The use of exogenous melatonin as a therapeutic modality in patients with postoperative delirium has been mentioned in only one report consisting of two cases. ${ }^{6}$ One case of antipsychotic/benzodiazepine-resistant postoperative delirium was successfully treated with $2 \mathrm{mg}$ of a 
slow-release melatonin formulation. In the other case, a prophylactic dose of melatonin $2 \mathrm{mg}$ po qhs was administered for three postoperative nights to a patient who had a history of postoperative delirium. In this instance, the patient did not develop postoperative delirium, and he remained oriented throughout his five-day period of hospitalization. However, in an observational study involving 32 intensive care unit patients with tracheotomy, exogenous melatonin $3 \mathrm{mg}$ failed to increase nocturnal sleep or to reduce agitation or haloperidol consumption. $^{7}$

The clinical implications of the limited data and published clinical experience with melatonin suggest that large-scale studies are warranted to determine the potential therapeutic value of melatonin in managing patients at risk of developing postoperative delirium.

Competing interests None declared.

\section{References}

1. Dispersyn G, Pain L, Challet E, Touitou Y. General anesthetics effects on circadian temporal structure: an update. Chronobiol Int 2008; 25: 835-50.

2. Mukai M, Uchimura N, Hirano T, Ohshima H, Ohshima M, Nakamura J. Circadian rhythms of hormone concentrations in alcohol withdrawal. Psychiatry Clin Neurosci 1998; 52: 238-40.

3. Shigeta H, Yasui A, Nimura Y, et al. Postoperative delirium and melatonin levels in elderly patients. Am J Surg 2001; 182: 449-54.

4. Miyazaki T, Kuwano H, Kato H, et al. Correlation between serum melatonin circadian rhythm and intensive care unit psychosis after thoracic esophagectomy. Surgery 2003; 133: 662-8.

5. Balan S, Leibovitz A, Zila SO, et al. The relation between the clinical subtypes of delirium and the urinary level of 6-SMT. J Neuropsychiatry Clin Neurosci 2003; 15: 363-6.

6. Hanania M, Kitain E. Melatonin for treatment and prevention of postoperative delirium. Anesth Analg 2002; 94: 338-9.

7. Ibrahim MG, Bellomo R, Hart GK, et al. A double-blind placebocontrolled randomised pilot study of nocturnal melatonin in tracheostomised patients. Crit Care Resusc 2006; 8: 187-91. 